\title{
What ENT doctors should know about COVID-19 contagion risks
}

\author{
Giuseppe Meccariello ${ }^{1}$ and Oreste Gallo ${ }^{2}$ \\ ${ }^{1}$ Department of Head-Neck Surgery, Otolaryngology, Head-Neck and Oral Surgery Unit, \\ Morgagni Pierantoni Hospital, Azienda USL della Romagna, Forlì, Italy \\ ${ }^{2}$ Head, ENT clinic, Department of Experimental and Clinical Medicine, University of \\ Florence, Florence, Italy
}

April 28, 2020

\section{Updates}

A general consensus exists on coronavirus diffusion by droplet transmission, especially the aerosolisation during hospital procedures like intubation or bronchoscopy might represent a big concern, exposing other patients and health-care staff to an increased risk of infection In this context, the general otolaryngology procedures may determine an aerosolisation with nosocomial amplification of the infection.

In particular flexible and/or rigid nasolaryngoscopy may include some maneuvers such as puffing out your cheeks, talking, swallowing some coloured water or poking out your tongue. Further, the introduction of the endoscope may cause sneezing and cough.

These risks can increase when in-office surgical procedures are applied to cure urgent and emergent pathologies such as epistaxis, removal of foreign bodies in upper aero-digestive tract, cricothyroidotomy as well as elective procedures such as biopsies, inferior turbinoplasty etc.

Based on the available evidence, it appears that SARS-CoV-2 can be transmitted by asymptomatic carriers, which contributes to its basic reproduction number and pandemic potential ${ }^{1}$.

Zou et $\mathrm{al}^{2}$ showed higher viral loads after symptom onset, with higher viral loads detected in the nose than in the throat. Further in the asymptomatic patients, the viral load was similar to symptomatic patients, which suggests the transmission potential of asymptomatic or minimally symptomatic patients.

The common work-load of a ENT are symptoms related to upper airways inflammations or infections. Sore throat with or without fever, sneezing, hoarseness may be prodromic symptoms of a COVID-19 infection in the incubation period ${ }^{3}$. Moreover, the coughing patients with a negative chest X-ray is one of the most consultation required.

Direct contact of droplet spray produced by coughing, sneezing or talking involves relatively large droplets containing organisms and requires close contact usually within $1 \mathrm{~m}^{4}$. Indirect contact may take place after the droplets are removed from the air by surface deposition ${ }^{5}$.

Han et $\mathrm{al}^{6}$ studied the dynamic features of bio-aerosolisation by sneezing. The velocity of the airflow exhaled by sneeze is much larger than that of breath and cough. Moreover, the total number of droplets generated during sneeze is also larger than that of other respiratory activities. According to the study on flow dynamics and characterization of cough, the maximum velocity of exhaled airflow can be found at $\mathrm{t}=57-110 \mathrm{~ms}$ for different persons which is most likely to occur at $100 \mathrm{~ms}$. Usually, sneeze lasts $0.3-0.7 \mathrm{~s}$, so $\mathrm{t}=100 \mathrm{~ms}$ is in the duration of the sneeze. As the velocity of the airflow exhaled by sneeze is really high, it can be assumed that the droplets that are exhaled at $\mathrm{t}=0-100 \mathrm{~ms}$ will not re-enter the measurement zone before 
$\mathrm{t}=100 \mathrm{~ms}$. The high-speed airflow and corresponding turbulence produced by sneeze may also lead to a large number of droplets, i.e. the number of the droplets generated by sneeze is about 18 times larger than that of cough. Further, the size of sneezing droplets is 341.5-398.1 $\mu \mathrm{m}$ for unimodal distribution and 73.6-85.8 $\mu \mathrm{m}$ for bimodal distribution. After the droplets are exhaled into the indoor environment, the evaporation effects will strongly influence the size and mass of the droplets. The final equilibrium diameter of expiratory droplets after evaporation is highly dependent upon the temperature and relative humidity of the environment. In the indoor environment, the relative humidity and temperature are much lower than those in the respiratory tract. So the volatile content of these droplets will keep evaporating and result in the shrinkage of the droplets.

Definitively, these findings demonstrate that the routine activities of an otolaryngologist are constantly at high risk of contagion in COVID-19 epidemic areas.

Taking a look at the current Italian situation, the experience of the region Veneto demonstrated that the application of COVID-19 screening also in asymptomatic people can reduce the contagion spreading. Thus, it seems clear that extend the screening to all health-workers included otolaryngologists could be a valid strategy to reduce the onset of a worst case scenario, the hospital outbreak.

In conclusion, the professional exposure to SARS-CoV-2 is really high for the otolaryngologist and nurse staff, even in in-office settings. Personal protective equipments are strongly recommended as well as for health-workers in close contact with infected patients.

\section{REFERENCES}

Zhu W, Xie K, Lu H, Xu L, Zhou S, Fang S. Initial clinical features of suspected Coronavirus Disease 2019 in two emergency departments outside of Hubei, China. J Med Virol. 2020 Mar 13. doi: 10.1002/jmv.25763. [Epub ahead of print]

Zou L, Ruan F, Huang M et al. SARS-CoV-2 Viral Load in Upper Respiratory Specimens of Infected Patients. N Engl J Med. 2020 Feb 19. doi: 10.1056/NEJMc2001737. [Epub ahead of print]

Lauer SA, Grantz KH, Bi Q et al. The Incubation Period of Coronavirus Disease 2019 (COVID-19) From Publicly Reported Confirmed Cases: Estimation and Application. Ann Intern Med. 2020 Mar 10. doi: 10.7326/M20-0504. [Epub ahead of print]

Leder K, Newman D. Respiratory infections during air travel. Intern Med J. 2005 Jan;35(1):50-5.

Chao CYH, Wan MP, Sze To GN. Transport and removal of expiratory droplets in hospital ward environment. Aerosol Sci Technol 2008;42, 377 - 394.

Han ZY, Weng WG, Huang QY. Characterizations of particle size distribution of the droplets exhaled by sneeze. J R Soc Interface. 2013 Sep 11;10(88):20130560. 